\title{
Robust State Estimation Based on Orthogonal Methods and Maximum Correntropy Criterion
}

\author{
Victor Freitas Antonio Simões Costa \\ Federal University of Santa Catarina \\ Florianópolis, SC, Brazil \\ Emails: victor.silva@posgrad.ufsc.br, simoes.costa@ufsc.br
}

\author{
Vladimiro Miranda \\ INESC TEC and University of Porto \\ Porto, Portugal \\ Email:vmiranda@inesctec.pt
}

\begin{abstract}
This paper presents an orthogonal implementation for power system state estimators based on the Maximum Correntropy Criterion (MCC). The proposed approach leads to a numerically robust estimator which exhibits self-healing properties, in the sense that gross errors in analog measurements are automatically rejected. As a consequence, robust estimates are produced without the need of running the state estimator again after bad data identification and removal. Numerical robustness is achieved by means of a specialized orthogonal algorithm based on fast Givens Rotations, which is able to handle the dynamic measurement weighting mechanism implied by the Parzen window concept associated to MCC. Results for a 3-bus test system are presented to properly illustrate the Correntropy principles, and several case studies conducted on the IEEE 30-bus and 57-bus benchmark systems are used to validate the proposed methodology.
\end{abstract}

Index Terms-Maximum Correntropy Criterion, Orthogonal Algorithms, Real Time Power System Modeling, Robust State Estimation.

\section{INTRODUCTION}

The classic solution for Power System State Estimation (PSSE) provided by the Weighted Least Squares (WLS) method provides satisfactory results as long as the measurements processed by the estimator are free of gross errors. On the other hand, the presence of any bad data will severely contaminate the state estimates. It is therefore essential that conventional estimators be equipped with bad data processing capabilities to be executed as a post-processing stage. In case the presence of gross errors is detected, the erroneous measurements must be identified and removed, and state estimation must be restarted in order to obtain bad data-free estimates. Depending on the particular situation, the whole procedure may demand a significant amount of computational effort and computing time, what is clearly undesirable considering the strict time limits to which PSSE is subject to in a real-time environment.

The sensitivity of WLS solutions to bad data in PSSE is well known and has motivated research efforts for alternative, more robust PSSE methods. Accordingly, the use of non-quadratic criteria has been proposed in order to automatically reject bad data and provide good state estimates even when they are present [1], [2]. However, those earlier efforts lacked a solid theoretic basis to properly define the new estimation criteria. In the nineties, other authors have explored robust statistics concepts to derive non-quadratic estimators exhibiting more solid theoretical background [3], [4].

More recent efforts to improve the robustness of PSSE methods draw inspiration from contemporary developments in the Information Theory field [5], [6], leading to a new generation of state estimation algorithms based on the Maximum Correntropy Criterion (MCC) [7], [8]. In addition to being particularly resilient to the presence of bad data, the accessory use of the Parzen window concept [9] enables a controlled progression of the iterative process in order to properly handle multiple solution difficulties that are always a matter of concern with non-quadratic criteria.

Currently, solution methods based on the MCC approach are based on variants of the numerically fragile GaussNewton method [8] or metaheuristics techniques [7]. The main contribution of this paper is to develop an orthogonal solution for the maximum correntropy approach. Our purpose is twofold: to impart numerical robustness to the method, as well as to take advantage of the row-processing feature of fast Givens orthogonal rotations to better implement the variable measurement weighting mechanism inherent to the MCC approach.

This paper is organized as follows. Section II briefly reviews the background of MCC-based state estimation. The proposed solution through fast Givens Rotations is dealt with in Section III. The Parzen windows adjustment mechanism for the proposed approach is detailed in Section IV. Section $\mathrm{V}$ describes the solution algorithm. Illustrative results based on the 3-bus test system and statistical analysis of several simulations conducted with the IEEE 30-bus and 57-bus test systems are presented in Section VI. Section VII summarizes the main conclusions.

\section{PSSE BASED ON MAXIMUM CORRENTROPY CRITERION}

\section{A. PSSE modeling, Parzen Windows and Correntropy}

Power system state estimation is aimed at determining the optimal (in some pre-defined sense) solution to a system of overdetermined and inconsistent set of nonlinear equations given by:

$$
z=h(x)+\varepsilon
$$

where $\boldsymbol{x}$ is the $n \times 1$ state vector composed by bus voltage magnitudes and phase angles, and $\boldsymbol{z}, \boldsymbol{h}(\cdot)$ and $\varepsilon$ are $m \times 1$ vectors containing the measurements, nonlinear functions relating measured quantities and states, and measurement errors, respectively. In the absence of bad data, $\varepsilon$ is assumed to be normally distributed, zero mean, and uncorrelated, with known variances which depend on meter accuracies.

The conventional approach for PSSE is based on the WLS method, that minimizes the weighted sum of squared estimation residuals [10]. The maximum correntropy criterion, on 
the other hand, aims at extracting the maximum amount of information from residual distribution related to the available measurement set [5], [7]. This is accomplished through the Correntropy concept, which measures the similarity between measured and estimated values within a given "observation window". The latter is defined on the basis of the Parzen window technique, which makes use of a Gaussian kernel $\kappa_{\sigma}(\cdot)$, centered on each candidate solution point $\boldsymbol{x}^{k}$, and whose width is specified by the $\sigma$ parameter [9]. Such a kernel function for a particular measurement $i$ is defined as

$$
\kappa_{\sigma}\left(z_{i}-\hat{z}_{i}\right)=\frac{1}{\sqrt{2 \pi \sigma_{i}}} e^{-\frac{\left(z_{i}-\hat{z}_{i}\right)^{2}}{2 \sigma_{i}^{2}}}
$$

where $\hat{z}_{i}=h_{i}\left(\hat{\boldsymbol{x}}^{k}\right)$. By using (2), the Correntropy function $\mathcal{V}$ between the measurements contained in the whole vector $z$ and the corresponding estimated values of the measured quantities is given by:

$$
\mathcal{V}(\boldsymbol{z}, \hat{\boldsymbol{z}})=\frac{1}{m} \sum_{i=1}^{m} \kappa_{\sigma}\left(z_{i}-h_{i}(\hat{\boldsymbol{x}})\right)
$$

According to the MCC criterion, the state estimates are obtained by maximizing $\mathcal{V}(\boldsymbol{z}, \hat{\boldsymbol{z}})$. Using (3) and (2) and doing away with constant factors that have no effect on optimization, the MCC Problem can be stated as

$$
J_{M C C}=\max _{\hat{\boldsymbol{x}}} \sum_{i=1}^{m} e^{-\frac{\left(z_{i}-h_{i}(\hat{\boldsymbol{x}})\right)^{2}}{2 \sigma_{i}^{2}}}
$$

A desirable feature of Correntropy is that it is able to take into account all even moments of the residual function $\boldsymbol{z}-\boldsymbol{h}(\hat{\boldsymbol{x}})$ [5], depending on the width $\sigma$ of the Parzen window. Such parameter plays an important role in the iterative optimization process. For initially large $\sigma$ values, the MCC method is basically equivalent to the conventional WLS approach, since Correntropy reduces itself to the familiar Euclidean norm $\left(L_{2}\right)$ of the residuals. When $\sigma$ is progressively reduced during the iterative process, the Correntropy properties are gradually stressed, affecting mostly the residuals of large magnitudes. The net effect is to apply other types of less sensitive norms ( $L_{1}$ or even $L_{0}$ ) to them, thus substantially reducing their effect on the final state estimation solution.

By applying the optimality conditions to Problem (4), it is possible to show that the MCC solution can be obtained by solving the following linear system in each iteration:

$$
\boldsymbol{H}^{T} \boldsymbol{W} \boldsymbol{H} \cdot \boldsymbol{\Delta} \boldsymbol{x}=\boldsymbol{H}^{T} \boldsymbol{W} \boldsymbol{\Delta} \tilde{\boldsymbol{z}}
$$

where

$$
\begin{array}{lll}
\boldsymbol{W}=\boldsymbol{D}\left[\boldsymbol{I}-\boldsymbol{R}_{\boldsymbol{P}}\right] & ; & R_{P, i i}=\left(z_{i}-h_{i}\left(\hat{\boldsymbol{x}}^{k}\right)\right)^{2} / \sigma_{i}^{2} \\
\boldsymbol{\Delta} \tilde{\boldsymbol{z}}=\left[\boldsymbol{I}-\boldsymbol{R}_{\boldsymbol{P}}\right]^{-1} \boldsymbol{\Delta} \boldsymbol{z} & ; & D_{i i}=\frac{1}{\sigma_{i}^{2}} e^{-\left(z_{i}-h_{i}\left(\hat{\boldsymbol{x}}^{k}\right)\right)^{2} / 2 \sigma_{i}^{2}} .
\end{array}
$$

In the above equations, $\boldsymbol{H}$ is the Jacobian matrix of $\boldsymbol{h}(\cdot)$ calculated at a given point $\boldsymbol{x}^{k}, \boldsymbol{\Delta} \boldsymbol{z}=\boldsymbol{z}-\boldsymbol{h}\left(\boldsymbol{x}^{k}\right)$ and $\boldsymbol{I}$ is the $m \times m$ identity matrix.

As indicated in (6), measurement weighting matrix $W$ is dynamically updated through the iterations in order to implement the MCC principles, and this is the distinguishing feature of MCC with respect to the WLS approach. Matrix $\boldsymbol{W}$ must remain positive definite, and therefore care must be taken to prevent it to inadvertently become numerically indefinite in the course of the iterations. In the end of the each major iteration, state vector values are updated according to

$$
\boldsymbol{x}^{(k+1)}=\boldsymbol{x}^{k}+\boldsymbol{\Delta} \boldsymbol{x} \text {. }
$$

This process goes on until $\|\boldsymbol{\Delta} \boldsymbol{x}\|$ becomes smaller than a prespecified tolerance.

\section{Correntopy-Based State Estimation Solution THROUGH GIVENS ROTATIONS}

Orthogonal methods have been employed to provide numerically stable solutions for weighted least-squares problems in general [11], and have also been successfully applied to PSSE [12], [13]. They are particularly attractive in the current application where, as discussed in the previous section, measurement weights can significantly differ one from another. Employing weights whose values are widely apart is a known factor of numerical instability when solving equations whose form is similar to the Normal Equation, such as (5). Those arguments point out the importance of applying numerically robust algorithms to obtain the MCC solution.

In this paper, we propose the use of a fast, three-multiplier version of Givens rotations [11], hereafter referred to simply as $G 3 M$, instead of directly solving equation (5). It basically consists in successively applying orthogonal rotations to the rows of matrix $\boldsymbol{H}$ (augmented by incremental vector $\boldsymbol{\Delta} \tilde{\boldsymbol{z}}$ ) scaled by the MCC weights that compose the diagonal matrix $\boldsymbol{W}$ (see (6)). The original, 4-multiplier Givens rotations are aimed at eventually reducing the overdetermined linearized set of equations to a triangular system, whose easily obtained solution provides the state correction vector $\boldsymbol{\Delta} \boldsymbol{x}$ for that particular iteration. The whole process can be summarized as:

$$
\mathbf{Q}\left(\boldsymbol{W}^{\frac{1}{2}}[\boldsymbol{H} \mid \boldsymbol{\Delta} \tilde{\boldsymbol{z}}]\right)=\left[\begin{array}{l|l}
\mathbf{U} & \mathbf{c} \\
0 & \tilde{e}
\end{array}\right]
$$

where $\mathbf{Q}$ is the orthogonal matrix reflecting the cumulative effects of the rotations, $\mathbf{U}$ is the $n \times n$ upper triangular matrix, c is an $n \times 1$ vector and $\tilde{e}$ is a scalar.

The fast $G 3 M$ version of the rotations is based on factoring matrix $\mathbf{U}$ as [11], [12]:

$$
\mathbf{U}=\mathbf{T}^{\frac{1}{2}} \overline{\mathbf{U}}
$$

In (9), $\mathbf{T}$ is a diagonal matrix and $\overline{\mathbf{U}}$ is upper triangular with all diagonal entries equal to one. Since $\Delta \tilde{\boldsymbol{z}}$ is taken as an extra column of $\boldsymbol{H}$, vector $\mathbf{c}$ is also scaled accordingly and renamed $\overline{\mathbf{c}}$. In addition to requiring only three multiplications per rotations, the scaling artifice in (9) avoids costly square root computations, and that is why the $G 3 M$ method is often referred to as fast Givens rotations [11].

After the matrix reduction step is concluded, the incremental vector $\Delta \boldsymbol{x}$ is obtained by simply solving the triangular system

$$
\overline{\mathbf{U}} \Delta \boldsymbol{x}=\overline{\mathbf{c}}
$$

A desirable feature of applying $G 3 M$ to obtain the MCC solution is the ease with which the varying weights in $\boldsymbol{W}$ are taken into account during the process of applying the orthogonal rotations. Such property also facilitates the exclusion of 
detected outliers by simply skipping the corresponding rows of $\boldsymbol{H}$ (augmented by the associated measurement) during the process of applying the rotations.

\section{PARZEN Window Adjustment for the ORTHOGONAL MCC-ESTIMATOR}

As discussed in Section II, Correntropy properties are applied in conjunction with a procedure for Parzen window adjustment. The latter is aimed at determining in each MCC iteration the proper width of the Gaussian kernel within which the similarity between measurements and the corresponding estimated values is to be evaluated. That is to say, measurements whose errors are outside the window are virtually excluded from the estimation process. At the outset of the iterations, Parzen window widths are initialized at a value $\sigma^{0}$ sufficiently large to encompass all measurement errors. In the subsequent iterations, the window width $\sigma^{k}$ is gradually decreased in order to reject the effects of outliers possibly present in the measurement set. A lower bound is applied to $\sigma^{k}$ which depends on the assumed variances of the measurement errors in (1). The Parzen window adjustment policy should be such that whenever the available measurements are free from gross errors, the MCC solution is equivalent to the WLS solution.

It is possible to develop adjustment mechanisms such that a single parameter $\sigma$ is employed to all measurements, what amounts to adopting a unique "observation window" to all available measurements. However, better results can be obtained by using a Parzen window width to each measurement individually. In this paper, the proposed adjustment mechanism iteratively updates each Gaussian kernel size after computing (7). Accordingly, the kernel width for measurement $i$ in iteration $k, \sigma_{i}^{k}$, is updated as a function of the maximum normalized residual [10], weighted by the measurement standard deviation, that is

$$
\sigma_{i}^{k}=\sqrt{\Omega_{i i}} \cdot \max _{j}\left(\frac{\left|z_{j}-h_{j}(\boldsymbol{x})\right|}{\sqrt{\Omega_{j j}}}\right), \quad i, j \in\{1, \ldots, m\} \backslash \mathcal{S}
$$

where $\Omega$ is the residual covariance matrix, $\mathcal{S}$ is the set of outliers previously determined during estimation process, and "\" stands for the set difference operator. Therefore, rule (11) is applied only to measurements labeled as unsuspect up to that iteration, since those in $\mathcal{S}$ have already been discarded. It is important to emphasize that the maximum normalized residual test is not employed here as a post processing bad data identification procedure [10]. Rather, it is part of a strategy to update the Parzen window width in order to iteratively suppress bad data effects.

According to (11), the Gaussian kernel size value of each measurement decreases through the iterations until all $\sigma_{i}$ values are lower than a pre-specified threshold $\sigma_{m i n}$. As previously remarked, threshold $\sigma_{\min }$ must be larger than the standard deviation of measurement $i$ in order to avoid the undue exclusion of sound data from the estimation process.

\section{MCC-Estimator Algorithm}

This section presents the algorithm to solve the state estimation problem under the maximum Correntropy criterion. The solution of the proposed methodology approach, described in Sections II to IV, relies on the specialized orthogonal algorithm based on fast Givens rotations $(G 3 M)$. It is assumed that the available measurement set ensures system observability. The main steps of the algorithm are described below.

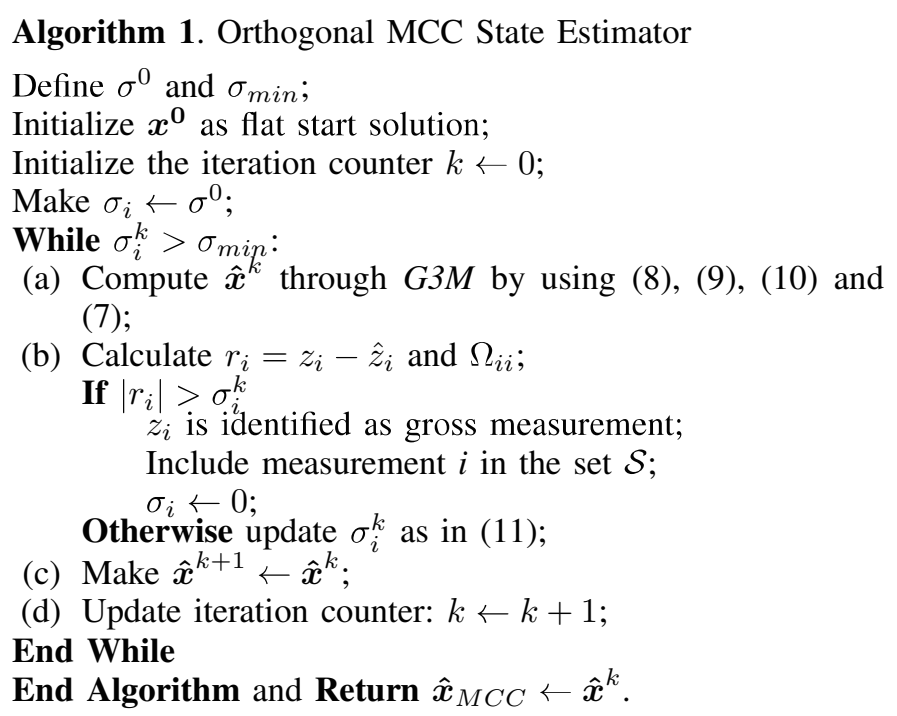

The following remarks should be made about some intrinsic characteristics of the proposed approach: $(i)$ the adopted Parzen window adjustment procedure establishes a threshold for the residual absolute value $\left|r_{i}\right|$ in each iteration, which is defined by its respective Gaussian kernel size $\sigma_{i}$. Therefore, when $\left|r_{i}\right|$ becomes larger than its own "observation window", measurement $z_{i}$ is identified as an outlier and included in set $\mathcal{S}$. The respective Gaussian kernel is then zeroed out, indicating that no further information is to be extracted from that measurement; (ii) to the benefit of computational efficiency, measurements included in bad data set $\mathcal{S}$ are not actually removed from the measurement vector. Instead, advantage is taken from the $G 3 M$ row-processing property. This enables a virtual measurement removal by checking the current values of the weights in matrix $\boldsymbol{W}$. Since the erroneous measurements violate the strictly positive weight restriction, outliers are simply skipped by the orthogonal technique; (iii) initialization based on arbitrary values (such as voltage flat start) is required only in the first iteration, since in subsequent ones current state estimates are used as initial state values.

The case studies reported in the following section illustrate the application of MCC-based state estimation.

\section{Simulation Results}

The proposed Orthogonal MCC-Estimator is evaluated through several case studies conducted with three test systems: a 3-bus test system, and the IEEE 30-bus and 57-bus networks. The first one illustrates the properties and main features of the MCC approach as compared with the WLS method, at a level of detail which is only feasible with a small system. The larger, more realistic power networks are employed to assess the performance of the proposed estimator under a multitude of scenarios that consider distinct load/generation conditions, as well as the presence of multiple bad data.

All simulations have been conducted in MATLAB using full nonlinear models for the test systems. A Gaussian random number generator is used to simulate the measurement errors, whose assumed accuracy levels are $3 \times 10^{-3}$ p.u. for 


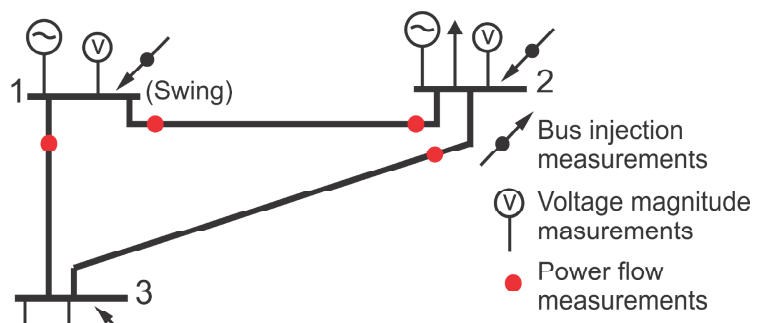

Fig. 1. 3-bus test-system

voltage magnitudes and $2 \times 10^{-2}$ p.u. for power flow and injection measurements. The metering schemes are composed of active/reactive power injection, active/reactive power flow, and bus voltage magnitude measurements, which are evenly distributed throughout the network. All power measurements are taken in active/reactive pairs.

\section{A. Illustrative results for the three-bus test system}

This subsection presents the results of simulations carried out with the 3-bus test system shown in Fig. 1. The voltage magnitude measurement at bus 2 is assumed to be a gross error, whose magnitude is equal to 15 standard deviations. Table I shows the actual values for measured quantities obtained from a power flow study, the measurement values, the estimates obtained from both conventional WLS method and the proposed MCC approach, and the corresponding residuals. For convenience, the data and the results for the gross measurement appear in bold face. It should be emphasized that no post-processing stage for handling bad data has taken place, so that all results have been obtained in the presence of the gross measurement for the two methods.

TABLE I

Measurement Estimates AND Residuals From WLS AND MCC ESTIMATORS

\begin{tabular}{cccccccc}
\hline \multirow{2}{*}{ Measur. } & Power & \multicolumn{2}{c}{ Measur. } & \multicolumn{2}{c}{ Measur. estimates } & & \multicolumn{2}{c}{ Residuals } \\
\cline { 5 - 6 } & Flow & Value & WLS & MCC & & WLS & MCC \\
\hline \hline$V_{1}$ & 1.0500 & 1.0412 & 1.1111 & 1.0473 & -0.0699 & -0.0062 \\
$\mathbf{V}_{\mathbf{2}}$ & $\mathbf{1 . 0 0 0 0}$ & $\mathbf{1 . 2 1 2 1}$ & $\mathbf{1 . 0 6 4 0}$ & $\mathbf{0 . 9 9 7 2}$ & $\mathbf{0 . 1 4 8 2}$ & $\mathbf{0 . 2 1 4 9}$ \\
$V_{3}$ & 0.9868 & 0.9889 & 1.0510 & 0.9838 & -0.0622 & 0.0051 \\
$p_{1}{ }_{2}$ & 0.7114 & 0.7102 & 0.7179 & 0.7165 & -0.0077 & -0.0063 \\
$p_{2}{ }_{1}$ & -0.7114 & -0.7161 & -0.7179 & -0.7165 & 0.0018 & 0.0004 \\
$p_{1}{ }_{3}$ & 0.8014 & 0.8117 & 0.8080 & 0.8094 & 0.0037 & 0.0023 \\
$p_{2} 3$ & 0.4114 & 0.4038 & 0.4165 & 0.4166 & -0.0127 & -0.0128 \\
$q_{1}{ }_{2}$ & 0.5271 & 0.5266 & 0.5205 & 0.5276 & 0.0061 & -0.0011 \\
$q_{2} 1$ & -0.4959 & -0.4914 & -0.5020 & -0.4951 & 0.0106 & 0.0037 \\
$q_{1}{ }_{3}$ & 0.3081 & 0.3095 & 0.3013 & 0.3102 & 0.0083 & -0.0007 \\
$q_{2} 3$ & 0.0319 & 0.0319 & 0.0304 & 0.0332 & 0.0015 & -0.0013 \\
$P_{1}$ & 1.5129 & 1.5276 & 1.5259 & 1.5259 & 0.0017 & 0.0017 \\
$P_{2}$ & -0.3001 & -0.3016 & -0.3014 & -0.2999 & -0.0002 & -0.0017 \\
$P_{3}$ & -1.2000 & -1.2141 & -1.2129 & -1.2128 & -0.0012 & -0.0013 \\
$Q_{1}$ & 0.8352 & 0.8391 & 0.8217 & 0.8379 & 0.0174 & 0.0013 \\
$Q_{2}$ & -0.4639 & -0.4639 & -0.4716 & -0.4619 & 0.0077 & -0.0021 \\
$Q_{3}$ & -0.2504 & -0.2512 & -0.2690 & -0.2490 & 0.0178 & -0.0022 \\
\hline
\end{tabular}

In order to have a global index to compare the performance of the two methods, we make use of a nodal voltage metric previously proposed in [14], given by:

$$
M_{V}=\left(\frac{1}{N} \sum_{i=1}^{N}\left|\vec{V}_{i}^{e s t}-\vec{V}_{i}^{t r u e}\right|^{2}\right)^{\frac{1}{2}}
$$

where $\vec{V}_{i}^{\text {est }}$ and $\vec{V}_{i}^{\text {true }}$ are the estimated and actual (obtained from the underlying power flow study) bus voltage phasors,
TABLE II

State Estimates Given By WLS AND MCC Estimators

\begin{tabular}{cccc|ccc}
\hline \multicolumn{4}{c|}{ Voltage Magnitudes } & \multicolumn{3}{c}{ Angles } \\
\cline { 2 - 7 } Bus & Power & & & Power & & \\
& Flow & WLS & MCC & Flow & WLS & MCC \\
\hline \hline 1 & 1.0500 & 1.1111 & 1.0473 & 0.0 & 0.0 & 0.0 \\
2 & 1.0000 & 1.0640 & 0.9972 & -0.0678 & -0.0608 & -0.0687 \\
3 & 0.9868 & 1.0510 & 0.9838 & -0.1505 & -0.1346 & -0.1529 \\
\hline
\end{tabular}

TABLE III

Bus Voltage Metric Values for WLS and MCC Estimators

\begin{tabular}{cc|c}
\cline { 2 - 3 } & WLS & MCC \\
\hline \hline$M_{V}$ & 0.0639 & 0.0032 \\
\hline
\end{tabular}

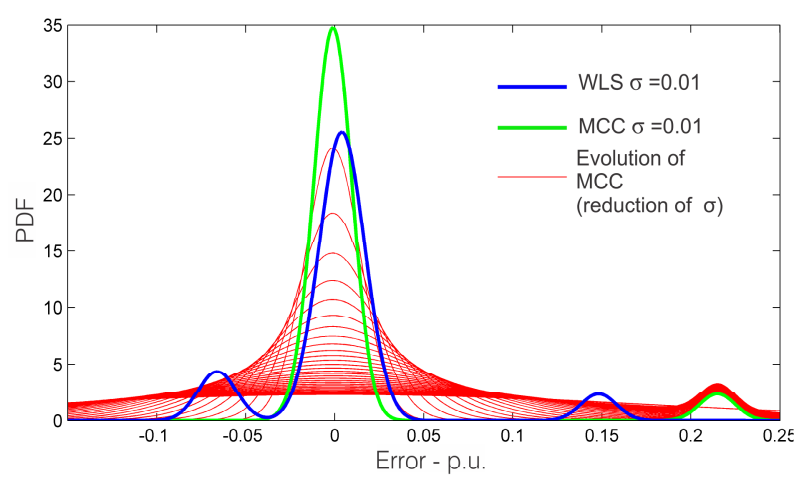

Fig. 2. Evolution of the error PDF through the Parzen windowing process for the MCC estimator and the error PDF of the WLS estimator

respectively. The metric values presented in Table III are obtained by applying the performance index defined in (12) to the results in the Table II.

Results in Tables I, II and III confirm that the gross error on measurement $V_{2}$ severely affects the state estimates computed by the WLS method. On the other hand, the estimates provided by the MCC approach are virtually free from the erroneous measurement effects.

To look into that difference of performance in more detail, a gradual reduction of Parzen windows with a fixed decrement, that is, $\sigma_{i}^{k+1}=\sigma_{i}^{k}-\Delta \sigma$ with a fixed $\Delta \sigma$, has been implemented for MCC. This adjustment mechanism differs from the proposed one based on (11) and admittedly leads to a significantly slower convergence rate. It is used here only for illustration purposes, since it allows a better visualization of how the estimates for the residual probability density function evolve through the iterations. The latter are shown as the plots in red in Fig. 2. The same figure shows in blue the corresponding pdf estimate obtained with the WLS method. One can notice that the WLS pdf significantly departs from a Gaussian function profile due to the influence of the bad data. On the other hand, the green plot obtained from MCC for a kernel width $\sigma_{M C C}=0.01$ shows that most residual errors are concentrated near zero, while a distant and isolated outlier of magnitude +0.21 p.u. is clearly identified. This illustrates how the effects of gross errors can be effectively suppressed by the dynamic window width adjustment mechanism of the MCC approach. 


\section{B. Performance assessment of the orthogonal MCC estimator as applied to realistic power networks}

In this subsection, the Givens rotations-based MCC estimator is applied to two benchmark systems in order to perform an overall evaluation of its bad data rejection properties under a variety of operation conditions. The IEEE 30-bus and 57-bus benchmark systems and the corresponding metering schemes are presented in Figs. 3 and 4, respectively. Data for these networks are available in [15]. Prior to applying the MCCbased state estimator, numerous case studies based on such test systems are conceived for distinct generation and load profiles as follows:

(a) 8,000 distinct operating conditions are considered over a 24-hour period for both networks encompassing light, intermediate, and heavy loading levels (i.e. $70 \%$ to $100 \%$ of full load);

(b) Measurements are simulated according to the metering schemes shown in Figs. 3 and 4 by superposing Gaussian noise with variance of $3 \%$ to the true values of the measured quantities;

(c) The true values for measured quantities and state variables are obtained from power flow studies conducted on each test system for all considered loading conditions.

In addition, all above cases are subject to the presence of none, single or multiple bad data. Gross error magnitudes are between 10 to 25 standard deviations, and the number of bad data per simulation varies from 0 to 5 . Among the 8,000 performed simulations per test system, 500 are bad data-free and the remaining 7,500 include at least one gross measurement.

The overall results for each of the test systems are summarized in Table IV. In the table, a success (S) means that the proposed algorithm has been able to capture all measurements simulated with gross errors. Otherwise, the bad data processing is labeled as failed $(\mathrm{F})$. The results in Table IV show that for cases without gross errors and with a single gross measurement the algorithm accomplishes an $100 \%$ success rate, for both test systems. This also means that, under those conditions, the orthogonal MCC-based estimator is able to correctly estimate the state variables and thus properly reject the effects of a

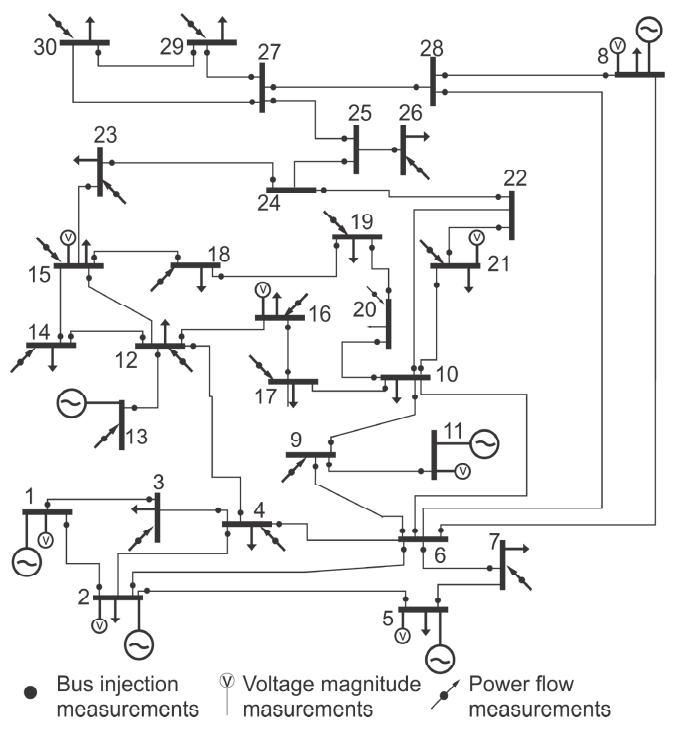

Fig. 3. IEEE 30-bus test system

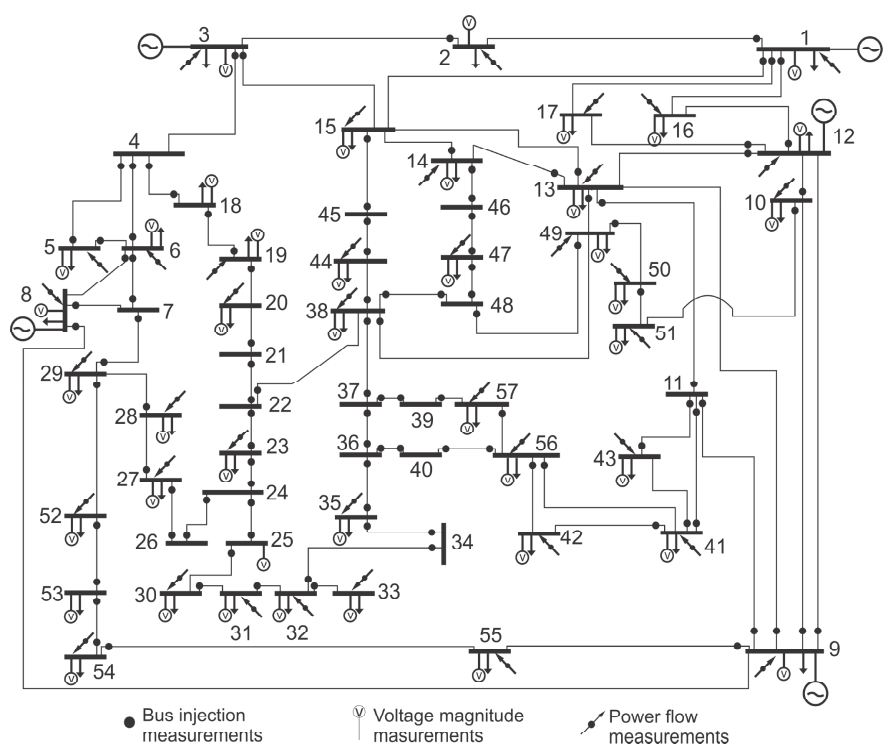

Fig. 4. IEEE 57-bus test system

TABLE IV

Algorithm EFfectiveness in the Presence OF 0-5 Gross ERrors

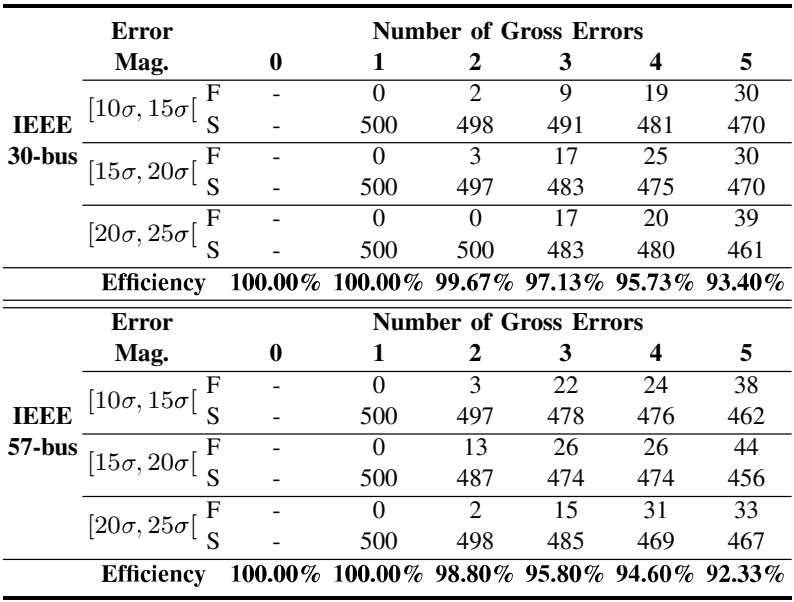

single bad data when they are present. For multiple bad data, some unsuccessful cases occur whose relative number grows slightly in proportion to the number of simulated outliers. Nevertheless, the efficiency levels are still high, since success rates of $93 \%$ and $92 \%$ are maintained even under the worst condition of 5 simultaneous gross errors.

Another factor to be evaluated is the quality of estimation results, which is defined here as the ability of the proposed approach to produce sufficiently accurate state estimates. The nodal voltage metric (12) is used for that purpose and applied to all successful cases presented in Table IV. A 0.01 p.u. threshold is employed to define the accepted accuracy level, that is, an estimator run is considered sufficiently accurate if $M_{V} \leq 0.01 \mathrm{p} . u$. The percentage rates of accurate results for the two test systems as a function of the number of simulated bad data are shown in Table V. Those results demonstrate that a remarkably high percentage of the state estimates are well adherent to the actual values.

Further investigation on the causes of the unsuccessful cases have been carried out. It reveals that most of those cases are due to the fact that the random sampling process used to select 
TABLE V

QuAlity of The Estimates FROM CASE STUdies With BAD DATA

\begin{tabular}{cccccc}
\hline Test & \multicolumn{5}{c}{ Number of Gross Errors } \\
System & $\mathbf{1}$ & $\mathbf{2}$ & $\mathbf{3}$ & $\mathbf{4}$ & $\mathbf{5}$ \\
\hline 30-bus & $100.00 \%$ & $98.92 \%$ & $97.64 \%$ & $98.03 \%$ & $96.76 \%$ \\
57-bus & $100.00 \%$ & $99.93 \%$ & $99.86 \%$ & $99.50 \%$ & $99.78 \%$ \\
\hline
\end{tabular}

TABLE VI

Overall EFFICIENCY CONSIDERING ONLY CASES Without OF CMs AND CSS

\begin{tabular}{cccccc}
\hline Test & \multicolumn{5}{c}{ Number of Gross Errors } \\
System & $\mathbf{1}$ & $\mathbf{2}$ & $\mathbf{3}$ & $\mathbf{4}$ & $\mathbf{5}$ \\
\hline 30-bus & $100.00 \%$ & $99.87 \%$ & $99.87 \%$ & $99.87 \%$ & $99.87 \%$ \\
57-bus & $100.00 \%$ & $99.93 \%$ & $99.80 \%$ & $99.87 \%$ & $99.73 \%$ \\
\hline
\end{tabular}

measurements with gross errors occasionally produces cases involving strongly correlated bad data. When processed by the algorithm of Section V, such cases may give rise to critical measurements (CMs) or critical sets of measurements (CSs) contaminated with gross errors. As it is well known from state estimation literature [10], [16], CMs and CSs pose severe challenges to any bad data processing method which should be resolved by measurement redundancy enhancement. If cases involving gross errors on CMs and/or CSs are disregarded, the success rates of the MCC-based estimator become superior to 99\% for the two test-systems, as shown in Table VI.

Finally, Table VII summarizes the convergence rates of the proposed algorithm. The table shows the average number of iterations for both the outer MCC loop and the inner $G 3 M$ loop. Clearly, the number of MCC iterations tends to increase with the bad data occurrences. As for the orthogonal solver based on Givens rotations, 2 to 3 iterations are typically required for convergence, regardless of how many gross errors are present.

TABLE VII

AVERAge CONVERGENCE RATES FOR THE ORTHOGONAL MCC-ESTIMATOR

\begin{tabular}{ccccccc}
\hline IEEE & Average & \multicolumn{5}{c}{ Number of Gross Errors } \\
System & Values & $\mathbf{1}$ & $\mathbf{2}$ & $\mathbf{3}$ & $\mathbf{4}$ & $\mathbf{5}$ \\
\hline \multirow{2}{*}{ 30-bus } & MCC iter. & 2.87 & 4.01 & 4.54 & 5.56 & 6.91 \\
& $G 3 M$ & 2.47 & 2.43 & 2.42 & 2.34 & 2.33 \\
\hline \multirow{2}{*}{ 57-bus } & MCC iter. & 2.92 & 3.83 & 4.80 & 5.80 & 6.76 \\
& $G 3 M$ & 2.30 & 2.34 & 2.32 & 2.30 & 2.26 \\
\hline
\end{tabular}

\section{CONCLUSIONS AND FinAl REMARKS}

This paper introduces an orthogonal implementation for a novel state estimation algorithm based on the maximum Correntropy principle. Correntropy fundamentals pertain to the field of Information Theory, and are based on the rationale of extracting maximum information from existing observation gathered from a given process. The accessory use of dynamically adjusted Parzen windows allows that outliers be screened and suppressed during the iterations, which makes the method resilient to the presence of bad data. On the other hand, the proposed orthogonal implementation imparts numerical robustness to the bad data suppression properties of Maximum Correntropy Criterion.

Simulations conducted on a small network illustrate the Correntropy properties as well as its robustness upon the presence of gross errors. The mechanism proposed in this paper for Parzen windows adjustment is tested through extensive simulations that include a variety of power system operating conditions and the occurrence of multiple bad data, by using two IEEE test systems. Reported results indicate remarkable high rates of successful results, both in terms of bad data suppression and final quality of the state estimates. The average convergence rates of the proposed algorithm are quite reasonable, particularly if one considers that the proposed estimator does away with the conventional post-processing stage for bad data identification and removal.

\section{ACKNOWLEDGMENT}

The authors gratefully acknowledge the support of the Brazilian National Research Council (CNPq) through Projects 312037/2013-9 (A. Simões Costa) and 400799/2014-6. V. Miranda also acknowledges the support of the European Regional Development Fund (FEDER) as well as the Foundation for Science and Technology (FCT) (ref. POCI-01-0145-FEDER016731).

\section{REFERENCES}

[1] H. M. Merrill and F. C. Schweppe, "Bad data suppression in power system static state estimation", IEEE Trans. on Apparatus and Systems, vol. PAS-90, no. 6, pp. 2718-2725, Nov. 1971.

[2] E. Handschin, F. C. Schweppe, J. Kohlas, and A. Fiechter, "Bad data analysis for power system state estimation", IEEE Trans. on Power Apparatus and Systems, vol. 94, no. 2, pp. 329-337, Mar. 1975

[3] L. Mili, H. G. Cheniae, N. S. Vichare, and P. J. Rousseeuw, "Robust State estimation based on projection statistic [of power systems]", IEEE Trans. on Power Systems, vol. 11, no. 2, pp. 1118-1127, May. 1996.

[4] R. C. Pires, A. Simões Costa, and L. Mili, "Iteratively reweighted least-squares state estimation through Givens rotations", IEEE Trans. on Power Systems, vol. 14, no. 4, pp. 1499-1507, Nov. 1999.

[5] W. Liu, P. P. Pokharel, and J. C. Principe, "Correntropy: Properties and Applications in Non-Gaussian Signal Processing", IEEE Transactions on Signal Processing, vol. 55, no. 11, pp. 5286-5298, Nov. 2007.

[6] D. Erdogmus, and J. C. Principe, "Generalized information potential criterion for adaptive system training", IEEE Trans. on Neural Networks, vol. 13, no. 5, pp. 1035-1044, Sep. 2002.

[7] J. K. Opara. "Information Theoretic State Estimation in Power Systems". PhD Thesis, University of Porto, Portugal, 2013

[8] W. Wu, Y. Guo, B. Zhang, A. Bose, and S. Hongbin, "Robust state estimation method based on maximum exponential square IET Generation, Transmission and Distribution, vol. 5, no. 11, pp. 1165 1172, May. 2011.

[9] E. Parzen, "On estimation of a probability density function and mode", Annals of Matlhematical Statistic, vol. 33, pp. 1065-1076, Sep. 1962.

[10] A. Monticelli, "Power System State Estimation: A Generalized Approach", Norwell, MA, USA: Kluwer, 1999.

[11] W. M. Gentleman, "Least squares computations by Givens transformations without square roots",Journal of the Inst. of Mathe. Applications, vol. 12, pp. 329-336, 1973.

[12] A. Simões Costa, and V. H. Quintana, "An orthogonal row processing algorithm for power system sequential state estimation", IEEE Trans. on Power Apparatus and Systems, vol. PAS-100, no. 8, pp. 3791-3800, Aug. 1981.

[13] N. Vempati, I. W. Slutsker, and W. F. Tinney, "Enhancements to Givens rotations for power system state estimation",IEEE Trans. on Power Systems, vol. 6, no. 2, pp. 842-849, May. 1991.

[14] KEMA, "Metrics for determining the impact of phasor measurements on power system state estimation", DRAFT. Eastern Interconnection Phasor Project, Jan. 2006.

[15] Power Systems Test Case Archive (Sep. 2010). [Online]. Available: http://www.ee.washington.edu/research/pstca/.

[16] K. A. Clements and P. W. Davis, "Multiple Bad Data Detectability and Identifiability, A Geometric Approach", in IEEE Power Engineering Review, vol. PER-6, no. 7, pp. 73-73, July 1986. 